\title{
Remote multi-function fire alarm system based on Internet of things
}

\author{
Lihui Wang ${ }^{\mathrm{a}}$, Shuai Zhao*, b, Jianqing Huang ${ }^{\mathrm{c}}$ and Jianyu $\mathrm{Ji}^{\mathrm{d}}$ \\ School of hai, nan University, Danzhou 571737, China; \\ a3206639232@qq.com, *Corresponding author \\ Email: b1398785121@qq.com, ${ }^{\mathrm{c}} 744873997 @ q q . c o m,{ }^{\mathrm{d}} 372517133 @ q q . c o m$
}

Keywords: fire, intelligent alarm, remote real-time monitoring

\begin{abstract}
This project uses MCU STC15W408AS (stable, energy saving, high speed), temperature sensor DS18B20 (cheap, high efficiency, stable), MQ2 resistance type semiconductor smog sensor (high stability, fast response and economy) and NRF24L01 wireless transmitting and receiving module (energy saving, small volume, reliable) as the main body to achieve concentration temperature data presentation, intelligent voice alarming and short distance wireless transmission. The whole system is safe, reliable, cheap, quick reaction and good performance.

This project uses the MCU STM32F103RCT6 as the main control chip, and use WIFI module ESP8266, wireless module NRF24L01 to make the gateway. Users can remotely check and control the related devices in real-time on smartphones or computers. We can also realize the functions of intelligent fire monitoring, remote fire extinguishing, cloud data storage through the third party server BigIOT.
\end{abstract}

\section{Introduction}

Fire is one of the most common natural disasters in the world today. It caused devastating damage to life, property and natural environment [1]. According to statistics, a large number of fires happen every day in the whole country and the whole world. Thousands of people are killed every day. Forest fires are up to about 200 thousand times a year [2], which brings immeasurable losses to forests. Recent years, there have been too many unpleasant fires around us. For example, the recent micro-blog heat launched accident the Greentown fire. Four fresh lives are passed away including three children. Try to think, If our alarm device is a little more advanced, a little more accurate, a little faster, how many lives we can save and how many families we can save. This firmed my faith to contribute in the fire alarm.

\section{Research status at home and abroad}

Currently on the market of fire alarm are separate [3], each room should install a mutual communication, it will only sound the horn when fire alarm lamp light, and cannot realize remote alarm, remote control, and cannot be used for building a wide range of wireless communication continuity.

Remote data monitoring based on ZigBee wireless sensor network proposed by Ma Hai is data - centered, and has the characteristic of self - organized, rapid deployment, 
and so on. It is suitable for some special occasions [4]. Tian Ya based on ZigBee wireless sensor network system design and implementation through Jennic JN512-MO wireless microcontroller module, design the network node hardware platform and analyzed the architecture of the ZigBee protocol and the specifications of each layer [5,6]. The above design proposes a more feasible networking method, which may not be used for fire alarm because of the technical reasons. This system puts forward the implementation method of fire alarm, and designs and makes wireless sensor node for fire alarm detection, and Internet access method for remote monitoring and control.

\section{System overall scheme}

The wireless sensor nodes use the main control chip to collect the temperature signals through the temperature sensor and convert them into digital signals. The single chip microcomputer identifies whether the alarm lights flicker or not. Then we use the wireless module to transmit the alarm information to other rooms, wireless modules, to achieve rapid and efficient alarm effect. The gateway aggregates the information uploading server of the wireless node in the area, analyzes the information transmitted by the server, and sends it to the target node to control the remote wireless nodes.

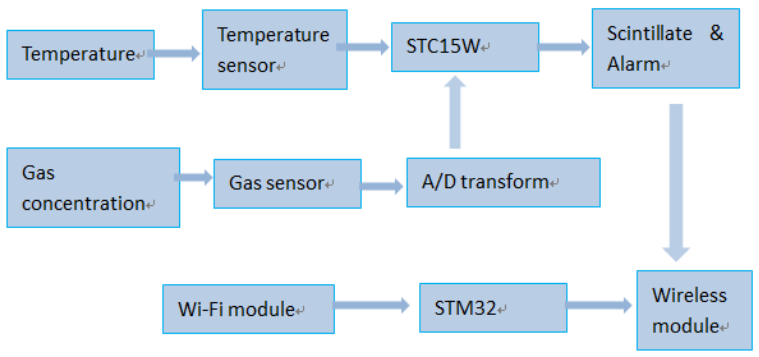

Figure 1 System flow

\subsection{Client for remote control}

Mobile client and computer web client can complete real-time detection of fire alarm data, real-time collection, real-time storage, and also can control any wireless sensor nodes by client. The specific client pages are shown in figures two and figure three.
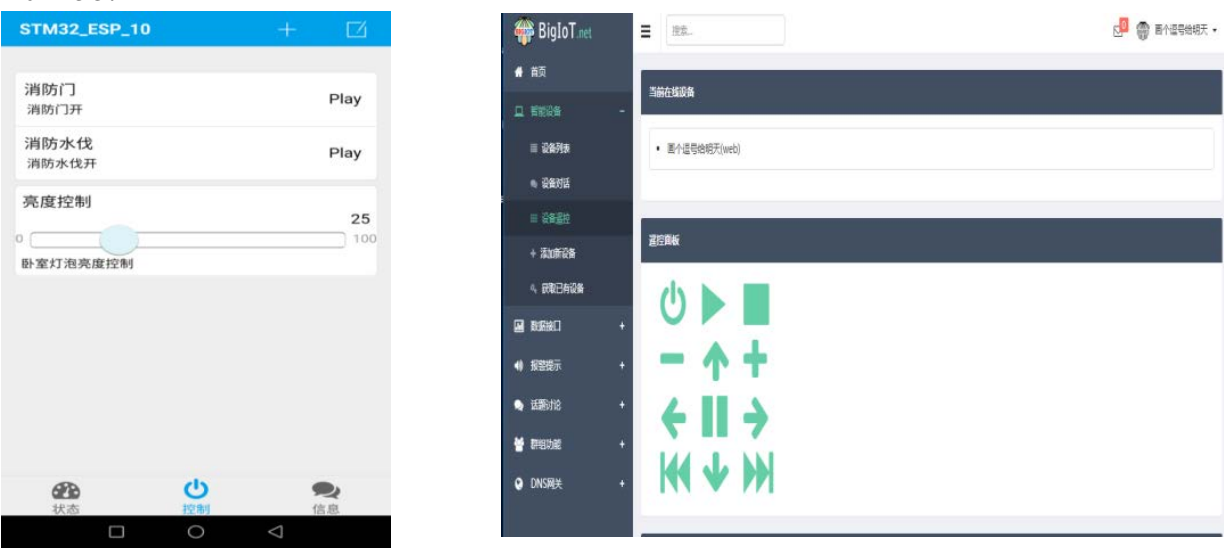

Figure 2 Client on Mobile Phone

Figure 3 Client on PC 


\subsection{Gateway}

This gateway learned from ZigBee's networking scheme and improved the ability of ad hoc network. The scheme can realize the networking of one thousand wireless sensor nodes, and achieve long distance wireless communication through relay nodes. Any node data can jump to any other node to transfer to the gateway at any time. The gateway can assign different channels to each node, and all the nodes will be encrypted with different passwords. [7]Even if a node is cracked, the data security of most of the nodes can still be guaranteed. Network data are transmitted by TCP network protocol and ensure that data is not missing and uploaded. The specific schematic diagram is shown in Figure 4.

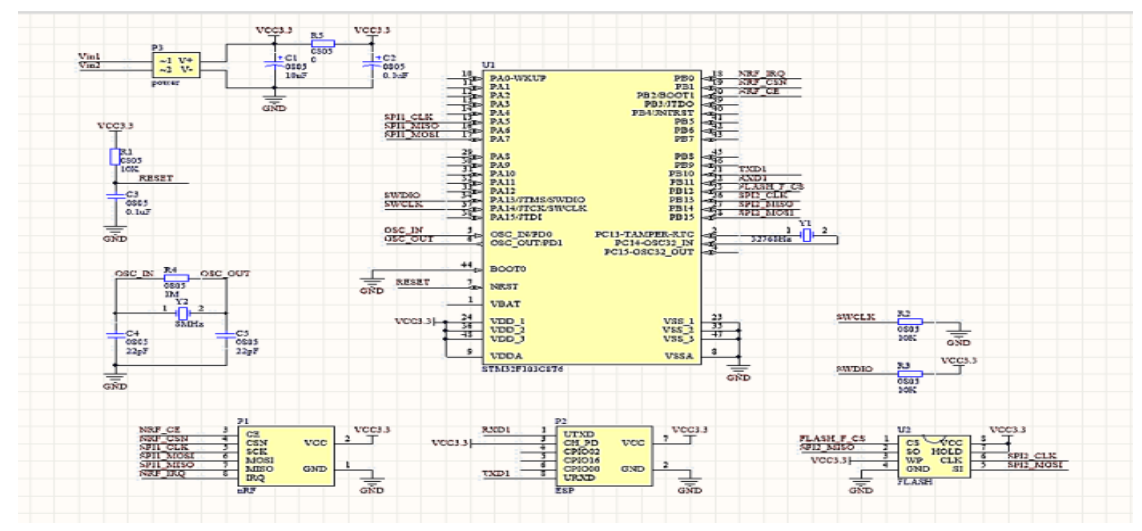

Figure 4 schematic of gateway

\subsection{Wireless sensor node}

Fire is a common natural phenomenon. Everyone knows that its essence is burning, so of course it needs combustibles, combustibles, and temperature. At the same time, aerosols are scientifically defined because the particles produced during the combustion process are suspended in the atmosphere. Some large particles produced during the formation of aerosol combustion are called smog. So the flame, aerosol, smoke, heat and so on during the whole process can be used as a fire parameter to determine whether a fire occurs or not. There are two kinds of danger in the fire (Flame, no Flame). Because no timely find no fire hazards, and combustible combustion of combustible gas, there is smoke, air contact burning, fire and generation of infrared radiation, ultraviolet radiation and generate a lot of heat, the rapid rise in ambient temperature. Incomplete combustion in the combustion process takes a lot of time, at this stage the temperature is not high, and there is a lot of smoke. In this project, fire alarm devices can be used to detect different fire parameters by inducing different fire parameters, and combined with high efficient, accurate and low cost targets. [8]The specific schematic diagram is shown in Figure five. 


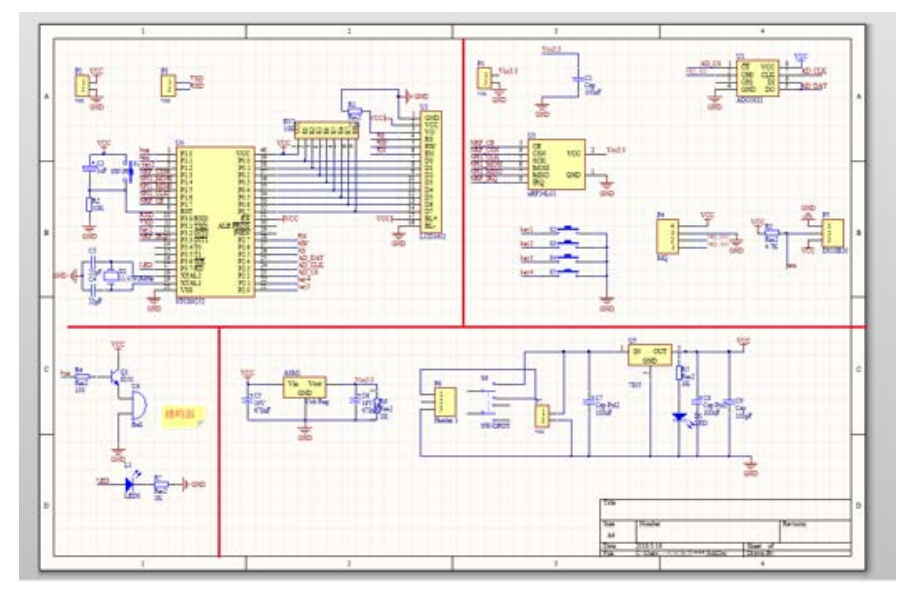

Figure 5 principle of wireless sensor nodes

\section{Conclusion}

The remote multi-function fire alarm system based on the Internet of things realizes the networking fire alarm, which is, deployable and family, and can also be deployed with large office buildings, and can also be deployed and used in the field for forest fire alarm. The nodes designed by the system are completely low power, and the two nodes 7 batteries can maintain the wireless node for one year. Using a packet jump transmission, the gateway can control all wireless nodes within one kilometer range.

\section{Acknowledgments}

2017 Special Higher Education Development Project of Hainan Province - Pilot Project of Electronic Science and Technology Transformation Professional Construction Project

\section{References}

1. Anping Niu. On the hazards of forest fires [J]. Economic and Technical Cooperation Information, 2009, (14): 98-98.

2. Qinghua Xin. CFAST simulation and safety evacuation of building fire [D]. Hunan: Central South University, 2009.

3. Yong Zhang.Discussion on Designing Idea of Fire Alarm System [J].Value Engineering, 2012, 31 (13):160-160.

4. Jing Jing, Beihai Li, Yue Yang, et al. Intelligent Building Technology [M]. Beijing: Science Press, 2008.

5. Limin Li, Ziyu Xuan, Lingyu Zhang. Microcontroller-controlled Fire Alarm Controller [J]. Automation Technology and Application, 2008, 27 (3): 88-90.

6. Design and Implementation of Data Monitoring System Based on Internet [J]. Lijie Han. Science and Technology Innovation Guide. 2010 (02).

7. ZigBee-based wireless sensor network node design [J]. Ren Zhang, Jianfeng Wang, Hai Yan. Mechanical and Electrical Engineering. 2008 (08)

8. Y.-K. Huang, A.-C. Pang, P.-C. Hsiu, W. Zhuang, P. Liu. Distributed throughput optimization for ZigBee cluster-tree networks. Parallel and Distributed Syst., IEEE Trans., 23 (2012), pp. 513-520. 